\title{
Therapeutic strategies for critically ill patients with COVID-19
}

\author{
Lei $\mathrm{Li}^{1 \dagger}$, Ranran $\mathrm{Li}^{1 \dagger}$, Zhixiong $\mathrm{Wu}^{2}$, Xianghong Yang ${ }^{3}$, Mingyan Zhao ${ }^{4}$, Jiao Liu ${ }^{5^{*}}$ and Dechang Chen ${ }^{1,5^{*}}$
}

\begin{abstract}
Since the 2019 novel coronavirus disease (COVID-19) outbreak originated from Wuhan, Hubei Province, China, at the end of 2019, it has become a clinical threat to the general population worldwide. Among people infected with the novel coronavirus (2019-nCoV), the intensive management of the critically ill patients in intensive care unit (ICU) needs substantial medical resource. In the present article, we have summarized the promising drugs, adjunctive agents, respiratory supportive strategies, as well as circulation management, multiple organ function monitoring and appropriate nutritional strategies for the treatment of COVID-19 in the ICU based on the previous experience of treating other viral infections and influenza. These treatments are referable before the vaccine and specific drugs are available for COVID-19.
\end{abstract}

Keywords: Coronavirus, 2019-nCoV, Antiviral therapy, Adjunctive intervention, Respiratory support

\section{Introduction}

In late December 2019, a group of patients with pneumonia of unknown cause were confirmed to be infected with a novel coronavirus (2019-nCoV) in Wuhan, China. The 2019-nCoV has now infected tens of thousands of people in China and has spread rapidly around the globe [1]. The World Health Organization (WHO) has declared coronavirus disease 2019 (COVID-19) as a Public Health Emergency of International Concern and released interim guidelines on patient management [2]. Due to the severity and the spreading of COVID-19 (novel coronavirus pneumonia, NCP), the Chinese government and the medical institutions have executed strict strategies to control the influence of this epidemic [3]. Until the end of February, the epidemic has been controlled to a great extent nationally. In Wuhan, the situation tends to be stable while a high proportion of critically ill patients are still under treatment of intensive care.

\footnotetext{
*Correspondence: catherine015@163.com; 18918520002@189.cn

${ }^{\dagger}$ Lei Li and Ranran Li contribute equally to this article

${ }^{5}$ Department of Critical Care Medicine, Ruijin North Hospital, Shanghai

Jiao Tong University School of Medicine, Shanghai 201800, People's

Republic of China

Full list of author information is available at the end of the article
}

\section{The identification of 2019-nCoV}

Coronaviruses (CoVs) are enveloped viruses with a single positive-stranded RNA genome ( $26-32 \mathrm{~kb}$ in length). $\mathrm{CoVs}$ mainly cause respiratory tract infections and some strains have high infectivity and mortality as well as heavy damage on public health, such as severe acute respiratory syndrome (SARS) and Middle East respiratory syndrome (MERS). The $2019-\mathrm{nCoV}$ is a $\beta-\mathrm{CoV}$ of group $2 \mathrm{~B}$ with over $70 \%$ similarity in genetic sequence with SARS-nCoV $[4,5]$. The latest version of diagnosis and treatment plan pointed out that the main transmission route is droplet transmission and close contact transmission. In addition, there are risks of airborne spread of 2019-nCoV during aerosol-generating medical procedures in specific circumstances $[6,7]$.

\section{Diagnosis and clinical classification of COVID-19}

The clinical manifestations of COVID-19 are fever, headache, dry cough, with radiological evidence of viral pneumonia. In severe cases, dyspnea usually occurs about 1 week after the disease onset and some patients can rapidly progress to acute respiratory distress syndrome (ARDS), septic shock, refractory metabolic acidosis, and coagulation disorders [8]. In addition, the clinical features of asymptomatic cases are not obvious except 
for the positive nucleic acid in oropharyngeal swabs. Asymptomatic cases also have the risk, although weak, of transmission. Respiratory viral infection can cause severe illness, especially in the elderly and persons with co-morbidities [9].

According to the latest version of diagnosis and treatment guidelines, confirmed cases infected with 2019$\mathrm{nCoV}$ are classified to have severe illness once complying with one of the following symptoms: (1) anhelation, respiratory rate $\geq 30$ times/min; (2) oxygen saturation at rest $\leq 93 \%$; (3) $\mathrm{PaO} 2 / \mathrm{FiO} 2 \leq 300 \mathrm{mmHg}$; and classified to be the critical/life-threatening illness once complying with one of the following symptoms: (1) respiratory failure, mechanical ventilation needed; (2) shock; (3) other organ dysfunction syndrome and requirement of intensive care unit admission.

The progress of the severe illness with COVID-19 is usually rapid and there is no clear separation between the severe illness and the critical illness. Therefore, patients of these two classes are combined to be the critical illness, which is helpful for health care workers to diagnose and treat patients with intensive care and resources at the early stage of the critical illness.

The diagnostic evidences for ICU admission according to the previous experience in the treatment of SARS include old age ( $>60$ years old), presence of co-morbidities (particularly, diabetes mellitus, hepatic or cardiac disease), and elevated lactate dehydrogenase levels on admission to hospitals $[10,11]$.

\section{Therapeutic strategies for COVID-19 in the ICU}

2019-nCoV invades through the respiratory mucosa and infects other cells, inducing cytokine storm systemically [12]. Some patients may progress rapidly with ARDS, disseminated intravascular coagulation (DIC), septic shock, and eventually multiple organ failure [13]. Therefore, early identification and timely treatment of critical cases is of crucial importance. Evidence-based therapy and supportive care in ICU is the mainstay for the management of severe and life-threatening illness of COVID-19.

The severe and critical illnesses with COVID-19 should be treated in ICU in the hospital with nosocomial infection control. Strict volume management, multi-organ function evaluation, critical care of the nutritional assessment/appropriate nutritional support are essential for these patients in ICU. In addition, attention should be paid to bedbound patients to prevent deep vein thrombosis.

\section{Antiviral therapy}

At present, there is no antiviral treatment with confirmed effectiveness for COVID-19. Available drug options that come from the clinical experience of treating SARS,
MERS and other previous influenza virus have been used for the treatment of COVID-19 patients. Although these antiviral drugs are promising in the treatment of COVID19, it should be kept In mind that: (1) the adverse effects of the drugs need to be monitored in clinic, (2) the effects of these drugs on critically ill patients still need to be clarified, (3) the potential mutation of the coronavirus may lead to the drug resistance of the virus.

\section{Fabiravir and ribavirin}

Nucleoside analogs have a broad-spectrum antiviral effect via the mechanisms of lethal mutagenesis, chain termination, and inhibition of nucleotide biosynthesis. Fabiravir and ribavirin are representative drugs of nucleoside analogs and exhibit the antiviral effect by inhibiting nucleotide biosynthesis. It has been demonstrated that the combination of fabiravir and oseltamivir in the treatment of severe influenza may accelerate clinical recovery than oseltamivir alone [14]. In addition, it has been reported that the combination of ribavirin and interferon alpha (IFN-a) significantly reduced the 14-day mortality of critically ill patients infected with MERS, although the 28-day mortality was not affected [15]. Ribavirin and IFN-a were also used in the treatment for SARS. However, ribavirin might have side effects such as anemia and liver injury, and IFN-a may not improve the patients' outcome [16]. Therefore, the use of ribavirin and IFN-a in the treatment of COVID-19 needs to be further elucidated by clinical studies.

\section{Lopinavir/ritonavir}

Lopinavir/ritonavir is a protease inhibitor in the treatment of HIV infection. Lopinavir/ritonavir showed the antiviral activity by inhibiting the replication of coronavirus in vitro. It has been reported that the combination of lopinavir/ritonavir with ribavirin could lower the risk of ARDS compared with ribavirin alone [17]. Most recently, the randomized clinical trial of lopinavir/ritonavir (400 mg/100 mg, twice-daily for 14 days) in the treatment of COVID-19 by Cao et al. has shown that in hospitalized adult patients with severe COVID-19, no beneficial effect was observed with lopinavir/ritonavir treatment compared with standard care group [18]. The adverse effects of lopinavir/ritonavir treatment include anorexia, nausea, abdominal discomfort, diarrhea, or acute gastritis. Moreover, the risk of hepatic injury, pancreatitis, more severe cutaneous eruptions, as well as the drug interactions due to CYP3A inhibition has been observed in the clinical trial, which arouses concern about the use of higher or prolonged dose regimens for outcome improvement [18]. In addition, serious complications such as acute kidney injury and secondary infection were fewer than in those not receiving treatment. Future trials with severe illness 
might help to elucidate the possibility of benefit of lopinavir/ritonavir treatment.

\section{Remdesivir}

Remdesivir (GS-5734) is a new nucleoside analog and has been recognized as a potential and promising antiviral drug against a wide array of RNA viruses, including SARS/MERS-CoV. It is currently under clinical development for the treatment of Ebola virus infection [19]. Remdesivir potentially inhibits the RNA-dependent RNA polymerase from MERS-CoV, reduces virus replication, decreases the virus titer in mouse lungs infected with MERS-CoV, and improves the lung tissue damage $[20,21]$. The antiviral activity of remdesivir and IFN-beta was found to be superior to that of the combination of lopinavir/ritonavir and IFN-beta against MERS-CoV [22]. A randomized, controlled trial has reported that the prolonged use of remdesivir in the treatment of Ebola virus disease (EVD) is safe [19], and no adverse events have been observed [23]. As a candidate drug that has not been approved, information about the side effects of remdesivir has not been reported yet. At present, two randomized, controlled, double-blind clinical trials are ongoing to evaluate the efficacy and safety of remdesivir (200 mg loading dose on Day 1, followed by $100 \mathrm{mg}$ i.v. once-daily maintenance dose for 9 days) in hospitalized patients with mild/moderate or severe COVID-19 respiratory disease $[24,25]$. The results of these clinical trials may open the window for effective antiviral therapy for such an epidemic infectious disease.

\section{Arbidol}

Arbidol is a small indole-derivative molecule and is approved for the prophylaxis and treatment of influenza and other respiratory viral infections [26]. It also showed inhibitory activity against other viruses, enveloped or not, responsible for emerging or globally prevalent infectious diseases such as hepatitis B and C [27]. In addition, arbidol has been reported to have antiviral activity against the pathogen of SARS, and the effect of arbidol mesylate-a derivative of arbidol, was almost five times higher than arbidol in reducing the reproduction of SARS in cells in vitro [28]. It has been claimed that arbidol was effective against 2019-nCoV in vitro [29]. A randomized multicenter controlled clinical trial of arbidol in patients with 2019-nCoV is in progress in China [30].

\section{Chloroquine and hydroxychloroquine}

It is known that angiotensin-converting enzyme-2 (ACE2) as a membrane protein is a functional receptor of SARS-CoV and it can facilitate virus entry into the cells by binding to the spike (S) protein of the virus, which mediates the fusion of viral and host membranes
[31-33]. Therefore, it may be of importance to block the binding of S protein to ACE2 to treat viral infection, such as SARS-CoV [34]. Chloroquine is a 9-aminoquinoline known since 1934, the sulfate and phosphate salts of which have both been commercialized as widely used antimalarial and autoimmune disease drugs. Chloroquine also shows broad-spectrum antiviral effects [35]. It was found to be a potent inhibitor of SARS-CoV infection due to its inhibitory effect on ACE2 [36]. It has been demonstrated that 2019-nCoV enter the epithelial cells of oral mucosa via the essential receptor ACE2 [37], and chloroquine can function at both entry and post-entry stages of 2019-nCoV infection [38]. Besides the antiviral activity, chloroquine has an immune-modulating activity, which may synergistically enhance its antiviral effect in vivo. Recently, Wang et al. have demonstrated that chloroquine is highly effective in the control of 2019$\mathrm{nCoV}$ infection in vitro and is suggested to be assessed in human patients suffering from COVID-19 [38]. In addition, the results from more than 100 COVID-19 patients have indicated that chloroquine phosphate is superior to the control treatment in inhibiting the exacerbation of pneumonia, improving lung imaging, promoting virus negative conversion, and shortening the disease course [39]. However, attention should be paid to the potential detrimental effects of chloroquine observed in previous attempts to treat viral diseases. At present, the clinical trials to evaluate the efficacy and safety of chloroquine in the treatment of COVID-19 is ongoing [40]. The use of chloroquine in the treatment of COVID-19 should refer to the most recent announcements if any.

In addition, hydroxychloroquine is a 4-aminoquinoline derivative antimalarial drug. Hydroxychloroquine is an immunosuppressive drug with mature clinical application in the treatment of rheumatic immune diseases such as rheumatoid arthritis and systemic lupus erythematosus $[41,42]$. It has been found to be more potent than chloroquine in inhibiting 2019-nCoV in vitro. Hydroxychloroquine sulfate $400 \mathrm{mg}$ given twice daily for 1 day, followed by $200 \mathrm{mg}$ twice daily for another 4 days is recommended in the treatment COVID-19 [43]. At present, the clinical evaluation of hydroxychloroquine in the treatment of COVID-19 is in progress [44], which might shortly provide preliminary results about the effectiveness of hydroxychloroquine.

\section{Antibacterial therapy}

Patients with pneumonia, especially in severe condition, may encounter with co-infection or cross-infection of bacterial pathogens, for instance staphylococcus aureus, during medical treatment in the hospital. Considering the high incidence of bacterial infection for critically ill patients with COVID-19, it is essential to test the kinetics 
of procalcitonin (PCT) and C-reaction protein (CRP) in COVID-19 patients for timely diagnosis and intervention of bacterial infection. According to the recent 2019 ATS/IDSA clinical practice guidelines, besides antiviral treatment for patients with viral-infected pneumonia, clinicians should empirically give antibacterial therapy to patients that initially have severe diseases (extensive pneumonia, respiratory failure, hypotension, and fever), or deteriorate after initial improvement, or fail to improve after 3 to 5 days of antiviral treatment [45]. Thus, antibiotic treatment is recommended in the treatment of COVID-19 patients based on the evidence of bacterial infection. The blind and inappropriate use of antibiotics, especially the broad-spectrum antibiotics, should be avoided.

\section{Adjunctive interventions}

Immune disorders have been observed in the treatment of patients with COVID-19. The virus infection activates the immune cells, leading to cytokine storm which is associated with disease severity [46]. On the other hand, the critical illness with COVID-19 mainly affect elders or people with chronic diseases, some of whom have very low number of lymphocytes, especially CD4+ T cells, implying deficiency of immune system. Therefore, to modulate the immune responses, a variety of pharmacologic agents have been proposed $[47,48]$.

\section{Corticosteroids}

The use of corticosteroids in the treatment of ARDS is controversial. Observational data in SARS suggest that immunomodulation with regimens of high-dose methylprednisolone might be helpful in modulating inflammatory responses and lung damage $[49,50]$. On the other hand, other studies showed that use of steroids is associated with increased risk for bacterial infection, increased mortality, and even antiviral resistance in influenza-associated pneumonia or ARDS [51-54]. Moreover, multiple studies have reported that corticosteroid treatment is associated with delayed viral shedding in hospitalized patients without significant change in 90-day mortality [55-57]. Corticosteroid therapy in patients with MERS was shown to be not associated with a difference in mortality, but associated with delayed MERS coronavirus RNA clearance [57].The early use of parenteral glucocorticoids therapy for fever reduction and pneumonia prevention has been shown to increase the risk for critical disease or death from H1N1 infection [58].

The currently limited clinical research does not support the use of corticosteroids in the treatment of ARDS in COVID-19 patients to improve the outcome of patients. The WHO recommended that corticosteroids should not be used in the treatment of viral pneumonia or ARDS.
There is no convincing proof for the therapeutic benefits of corticosteroids in the treatment of COVID-19, which still need to be demonstrated in clinical research.

\section{Thymosin alpha-1}

Thymosin alpha- 1 is a thymic peptide hormone with significant benefits in restoring the homeostasis of the host immune system [59]. It is chemically synthesized and used in diseases with impaired immune system [60]. It has been reported that the low lymphocyte count is associated with the poor prognosis of septic patients. The use of thymosin alpha-1 therapy in combination with conventional medical therapies was effective in improving clinical outcomes and reducing mortality in severe sepsis [61]. In addition, thymosin alpha- 1 can enhance the immune responses of SARS patients and help to limit the spreading of SARS [62]. Therefore, although there is no clinical evidence showing the beneficial effects of thymosin alpha-1 in COVID-2019, it has been recommended to be used for some patients to enhance cellular immunity for the resistance of viral infection.

\section{Cyclosporine A}

Cyclosporine $\mathrm{A}$ is widely used in transplantation and autoimmune disorders due to its immunosuppressive effect. Cyclophilin A as a key member of immunophilins is the cellular receptor for cyclosporine A [63]. The inhibition of cyclophilins by cyclosporine A could block the replication of coronavirus, including SARS-CoV [64]. Therefore, non-immunosuppressive derivatives of cyclosporine A might serve as broad-range $\mathrm{CoV}$ inhibitors applicable against emerging virus like 2019-nCoV, which still needs to be confirmed by clinical studies in the future.

\section{Interferons}

There are two types of interferons (IFNs), type I IFNs and type II IFNs. It has been demonstrated that type I IFNs can inhibit the replication of both SARS and MERS-CoV $[65,66]$. Kuri et al. have reported that IFN transcription was blocked in tissue cells infected with SARS-CoV and cells infected with SARS-CoV were able to partially restore their innate immune responsiveness to SARS-CoV after priming with small amounts of IFNs [67]. Moreover, in patients with severe MERS-CoV infection, the combination of IFN-alpha-2a with ribavirin was shown to improve survival [66]. Recently, the combination of remdesivir and IFN-beta was shown to have significant antiviral activity [22].

\section{Gammaglobulin}

Intravenous gammaglobulin is considered as the safest immunomodulating drug available for the treatment of 
severe infection and sepsis. It has high titers of neutralizing antibodies against broad-spectrum virus, bacteria, and other pathogens, and can modulate the host immune responses in several ways. However, a large-scale multicenter randomized placebo-controlled trial did not show improved survival with intravenous gammaglobulin in severe sepsis [68]. Moreover, a Cochrane review showed that intravenous gammaglobulin did not reduce the mortality of septic patients [69]. Therefore, there is no convincing argument to recommend intravenous gammaglobulin in the treatment of 2019-nCoV.

\section{Tocilizumab}

One of the most important mechanism underlying the deterioration of COVID-19 is cytokine storm characterized by elevated levels of IL6, IFN- and other cytokines, which will lead to ARDS or even multiple organ failure [70]. Tocilizumab is a recombinant humanized monoclonal antibody binding to IL6 receptor and inhibiting its signal transduction. Tocilizumab has been used in the treatment for rheumatoid arthritis (RA) [71]. Moreover, tocilizumab has been reported to be effective against cytokine release syndrome induced by CAR-T cell infusion against B cell acute lymphoblastic leukemia [72]. In Diagnosis and Treatment Guidelines of NCP (trial version 7.0) [73], tocilizumab is recommended for the immunotherapy of patients with extensive lung lesions and severe cases that show an increased level of IL6 in laboratory testing. The efficacy of tocilizumab in COVID19 patients still needs to be investigated.

\section{Chinese traditional medicine}

In 2003, Chinese traditional medicine was used to prevent and treat SARS [74]. In 2009, during the pandemic of H1N1 influenza, the Traditional Chinese Medicine of China issued a Chinese traditional medicine prevention program, which included several Chinese herbal medicine formulae for the prevention of infection of adults and children. ShuFengJieDu capsules and Lianhuaqingwen capsules have also played a role in the prevention and treatment of new respiratory infectious diseases such as influenza A (H1N1) $[75,76]$. Some studies have confirmed that Yupingfeng powder has antiviral, antiinflammatory and immunoregulatory effects [77]. A multicenter, large-scale, randomized trial found that Yinqiao powder plus another heat-clearing formula could reduce time for fever resolution in patients with the H1N1 influenza virus infection [78].

It is suggested that high-risk populations exposed to COVID-19 patients, including medical staff, family members, and other people who are in close contact with COVID-19 patients, as well as residents living in COVID-19 outbreak areas, might benefit from taking
Chinese traditional medicine formulae for prevention. However, the efficacy and safety of these Chinese traditional medicine formulae in COVID-19 need to be further confirmed by clinical trials.

\section{Convalescent plasma}

The convalescent plasma derived from the patients with antibodies against 2019-nCoV can be effective in reducing the mortality rate of critically ill patients with infectious disease [79]. Convalescent plasma has been found to have an immunotherapeutic potential for the treatment of MERS, SARS and Ebola virus disease [80-82]. The explanation for the efficacy of convalescent plasma therapy is that antibodies from convalescent plasma might suppress viremia via free viral clearance, blockade of new infection, as well as the acceleration of infected cell clearance [83]. In addition, the use of high-titer MERS serum from camel could significantly improve the histology of lung damage and increase the clearance of MERS-CoV in mice [84]. Moreover, the use of convalescent plasma or serum was also suggested by WHO under Blood Regulators network when vaccines and antiviral drug was unavailable for an emerging virus. Evidence shows that convalescent plasma therapy is not associated with the occurrence of severe adverse events [85]. Convalescent plasma, if available, can be used for the treatment of critically ill patients with COVID-19 after the evaluation of the valence of antibody. It is worthwhile to test the efficacy and safety of convalescent plasma transfusion in COVID-19 patients.

\section{Respiratory supportive strategies}

A meta-analysis showed that among patients with 2019$\mathrm{nCoV}$ infection, the incidence of ARDS is approximately $15 \%$ [12]. Moreover, between $50 \%$ to $85 \%$ of patients admitted to ICU have hypoxemia/or development of respiratory exhaustion [86]. Therefore, timely and effective respiratory support can contribute to reduce complications and improve the survival of such critically ill patients.

\section{Oxygen therapy and mechanical ventilation}

Oxygen therapy, high-flow nasal cannula, and non-invasive ventilation may reduce the need of endotracheal intubation and decrease ventilator-associated complications and mortality. However, several studies have reported that the failure of non-invasive ventilation was up to $85 \%$ in which invasive ventilation was ultimately required in the treatment of severe influenza A (H1N1) in Canada [87]. Non-invasive ventilation may be effective and safe for some patients, whereas it might increase virus transmission to health care workers because of risk for infected aerosol generation. Therefore, for the 
treatment of 2019-nCoV, non-invasive ventilation may be used in selected patients in early stages with milder acute hypoxemic respiratory failure [88]. While for critically ill patients, the effectiveness of transitionally oxygen therapy, such as respiratory status and oxygen index (PO2/ FiO2), needs to be closely monitored and it should be switched to mechanical ventilation when necessary.

High-flow nasal cannula has emerged as an alternative to non-invasive ventilation to prevent intubation and reduce mortality in patients with acute hypoxemic respiratory failure [89]. High-flow nasal cannula has been reported to significantly reduce 90 -day mortality of community-acquired pneumonia compared with standard oxygen or non-invasive ventilation [89]. Hui et al. have shown that the breath dispersion distance is limited therefore lowering the risk of air transmission. However, the loose connection of the cannula with nasal obstruction can significantly increase the dispersion distance [90]. Wearing masks (particularly N95) can effectively reduce the breath dispersion distance during high-flow nasal ventilation to prevent nosocomial transmission [91, 92]. In addition, high-flow nasal cannula might increase virus transmission risk due to aerosol generation. Therefore, staff protection of health care workers is critical.

Mechanical ventilation for patients with severe ARDS should be managed with lung-protective strategies to minimize ventilator-associated lung injury and to improve survival. The approach to minimize ventilatorassociated lung injury and to improve survival includes: ventilation with low tidal volumes ( 4 to $8 \mathrm{~mL} / \mathrm{kg}$ of predicted body weight), targeting plateau pressure $(<30$ $\mathrm{cmH}_{2} \mathrm{O}$ ) [93], and minimizing the inspired oxygen concentration to decrease oxygen toxicity. High positive endexpiratory pressure (PEEP) can reduce the need for high FiO2 by improving gas exchange and lung compliance, whereas too high PEEP may lead to lung overdistension and hemodynamic instability. Optimal PEEP which can be titrated by pressure-volume curve, oxygenation, stress index, electrical impedance tomography (EIT), ultrasound, and some other clinical parameters is associated with improved survival rate among severe ARDS patients [9].

Lung recruitment needs to be evaluated for mechanically ventilated patients when uncorrectable hypoxemia occurs, and lung recruitment should be executed for patients whose lungs can restore aeration. CT, EIT, ultrasound, and other bedside techniques should be used to evaluate lung recruitability before lung recruitment.

For critically ill patients managed with mechanical ventilation, excessive spontaneous breathing due to stretching may lead to lung injury. Therefore, neuromuscular relaxant may be used to control the spontaneous breathing and protect the lung.

\section{Prone positioning ventilation}

Prone positioning ventilation is a technique that often improves oxygenation in ARDS, possibly through improvements in ventilation-perfusion matching, the uniformity of ventilation, and gravity-related atelectasis. Prone ventilation was used in mechanically ventilated SARS patients without enough data to draw any conclusion with regard to its efficacy [86]. Although prone ventilation showed no improvement in survival or organ dysfunction overall, it might be beneficial for patients with severe ARDS. A multicenter RCT demonstrated that early application of prone positioning in patients with severe ARDS resulted in decreased mortality [94]. In addition, the use of prone positioning in patients with H7N9 influenza-induced severe ARDS was shown to be related with improved oxygenation, sustained after returning to a supine position, and with decreased carbon dioxide retention [95]. Generally speaking, prone positioning ventilation for no less than $12 \mathrm{~h}$ daily is a relatively safe procedure that rarely worsens a patient's respiratory status. It can be thus recommended for the treatment of 2019-nCoV-induced severe ARDS.

\section{Extracorporeal membrane oxygenation (ECMO)}

ECMO has become the important life-support strategy for the standardized treatment of ARDS patients. ECMO should be considered as early as possible when the lung recruitment and prone positioning ventilation show to be ineffective.

Observational studies have reported that patients with ARDS induced by H1N1 influenza showed lower hospital mortality with transfer to an ECMO center compared with matched non-ECMO-supported patients [96]. The use of ECMO also showed survival benefit in patients with severe MERS [97]. ECMO tends to improve patient outcomes when used among those with limited organ failures and good pre-morbid functional status [9]. Substantial proportion of critically ill patients with COVID19 appear to have developed cardiac arrhythmias or shock [13], and may need ECMO support. However, for those who will develop septic shock or refractory multiple organ failure, ECMO is not suggested due to its less benefit.

ECMO is a resource-intensive, highly specialized and expensive form of life support with potential for significant complications such as hemorrhage and nosocomial infection. Therefore, the use of ECMO should be strictly limited in the treatment of COVID-19. Moreover, since the number of critically ill patients is still increasing and the resource of ECMO is finite, judgment is needed to decide when ECMO may be worthwhile and when it may not. Support with ECMO is supposed to be for the most 
critically ill patients in regions with extensive resources for this therapy [98].

\section{Circulatory support and fluid management}

For patients with ARDS, restrictive and timely fluid resuscitation is associated with better oxygenation and lower mortality. Aggressive fluid administration may worsen oxygenation and ventricular dysfunction, which may result in longer duration of mechanical ventilation and even mortality. Therefore, it is necessary to assess fluid responsiveness and to evaluate ventricular function during fluid resuscitation. Conservative fluid administration while maintaining adequate mean arterial pressure and organ perfusion with the appropriate use of diuretics and vasopressors is of importance [99].

\section{Multiple organ functional support and nutrition strategy}

According to the latest epidemiological report, the incidence for the critically ill patients to develop multiple organ dysfunction syndrome is up to $11 \%$ [48]. COVID19 may be combined with other organ injuries, including liver injury, cardiac dysfunction, coagulopathy, which may need the routine functional support for critically ill patients in ICU.

Moreover, all the critically ill patients with COVID19 admitted into ICU have negative nitrogen balance and malnutrition [12, 46], which has been considered as a contributing factor to the emergence of viral infectious diseases. Therefore, appropriate nutritional strategy is pivotal for the treatment of critical illnesses when necessary.

\section{Summary}

There are no specific antiviral drugs or vaccines for 2019$\mathrm{nCoV}$ at present. Therefore, it is important to enhance the host immune response against the infection with 2019-nCoV. All of the drug options are based on the experience treating SARS, MERS or some other previous influenza viruses. The efficacy of existing drugs as well as adjunctive pharmacologic interventions in the treatment of critical ill patients with COVID-19 warrants further verification in clinical research. To completely stop the epidemic spreading of COVID-19, a vaccine for 2019$\mathrm{nCoV}$ is urgently needed. Besides enhancing the host immune responses against viral infection, appropriately respiratory supportive strategies, monitoring and support of multiple organ function, modulating the immune status and inflammatory responses individually, as well as the prophylaxis and treatment of complications are all important guarantee for the recovery of critically ill patients with COVID-19. For a better understanding of this novel virus, more research needs to be done to get optimal strategies for the treatment of COVID-19.

\begin{abstract}
Acknowledgements
The authors would like to express their appreciation for all of the health care workers and other hospital staff for their efforts in combating the outbreak of COVID-19.
\end{abstract}

\section{Authors' contributions}

All the authors have participated in literature retrieval and viewpoint discussion. $\mathrm{LL}$ and $\mathrm{RL}$ have written this article under the supervision of $\mathrm{DC}$ and JL. All authors read and approved the final manuscript.

\section{Funding \\ Not applicable.}

\section{Availability of data and materials}

Not applicable.

Ethics approval and consent to participate

Not applicable.

Consent for publication

The authors declare that they agree for the publication of this article.

\section{Competing interest}

The authors declare that they have no competing interests.

\section{Author details \\ ${ }^{1}$ Department of Critical Care Medicine, Ruijin Hospital, Shanghai Jiao Tong University School of Medicine, Shanghai 200025, People's Republic of China. ${ }^{2}$ Department of Surgical Intensive Care Unit, Huadong Hospital Affiliated to Fudan University, Shanghai 200040, People's Republic of China. ${ }^{3}$ Department of Critical Care Medicine, Zhejiang Provincial People's Hospital, Hangzhou, Zhejiang 310014, People's Republic of China. ${ }^{4}$ Department of Criti- cal Care Medicine, The First Hospital Affiliated to Harbin Medical University, Harbin 150001, People's Republic of China. ${ }^{5}$ Department of Critical Care Medi- cine, Ruijin North Hospital, Shanghai Jiao Tong University School of Medicine, Shanghai 201800, People's Republic of China.}

Received: 24 March 2020 Accepted: 6 April 2020

Published online: 20 April 2020

\section{References}

1. Zhu N, Zhang D, Wang W, et al. A novel coronavirus from patients with pneumonia in China, 2019. N Engl J Med. 2020;382(8):727-33.

2. Organization WH. clinical-management of severe acute respiratory infection when novel coronavirus (2019-nCoV) infection is suspected-interim guidance. Published January 28, 2020.

3. Qiu H, Tong Z, Ma P, et al. Intensive care during the coronavirus epidemic. Intensive Care Med. 2020. https://doi.org/10.1007/s00134-020-05966-y.

4. Hui DS, Azhar I, Madani TA, et al. The continuing 2019-nCoV epidemic threat of novel coronaviruses to global health - the latest, novel coronavirus outbreak in Wuhan, China. Int J Infect Dis. 2019;2020(91):264-6.

5. Lu R, Zhao X, Li J, et al. Genomic characterisation and epidemiology of 2019 novel coronavirus: implications for virus origins and receptor binding. Lancet. 2020;395(10224):565-74.

6. Yu YX, Sun L, Yao K, et al. Consideration and prevention for the aerosol transmission of 2019 novel coronavirus. Zhonghua Yan Ke Za Zhi. 2020;56:E008.

7. van Doremalen N, Bushmaker T, Morris DH, et al. Aerosol and surface stability of SARS-CoV-2 as compared with SARS-CoV-1. N Engl J Med. 2020. https://doi.org/10.1056/NEJMc2004973.

8. Wang Z, Chen X, Lu Y, et al. Clinical characteristics and therapeutic procedure for four cases with 2019 novel coronavirus pneumonia receiving combined Chinese and Western medicine treatment. Biosci Trends. 2020. https://doi.org/10.5582/bst.2020.01030. 
9. Arabi YM, Fowler R, Hayden FG. Critical care management of adults with community-acquired severe respiratory viral infection. Intens Care Med. 2020. https://doi.org/10.1007/s00134-020-05943-5.

10. Booth CM, Matukas LM, Tomlinson GA, et al. Clinical features and shortterm outcomes of 144 patients with SARS in the greater Toronto area. JAMA. 2003;289(21):2801-9.

11. Lee $\mathrm{N}$, Hui $\mathrm{D}, \mathrm{Wu}$ A, et al. A major outbreak of severe acute respiratory syndrome in Hong Kong. N Engl J Med. 2003;348(20):1986-94.

12. Sun P, Qie S, Liu Z, et al. Clinical characteristics of 50466 hospitalized patients with 2019-nCoV infection. J Med Virol. 2020. https://doi. org/10.1002/jmv.25735.

13. Wang D, Hu B, et al. Clinical characteristics of 138 hospitalized patients with 2019 novel coronavirus-infected pneumonia in Wuhan, China. JAMA. 2020. https://doi.org/10.1001/jama.2020.1585.

14. Wang Y, Fan G, Salam A, et al. Comparative effectiveness of combined favipiravir and oseltamivir therapy versus oseltamivir monotherapy in critically ill patients with influenza virus infection. J Infect Dis. 2019. https ://doi.org/10.1093/infdis/jiz656.

15. Kim UJ, Won EJ, Kee SJ, et al. Combination therapy with lopinavir/ritonavir, ribavirin and interferon-alpha for Middle East respiratory syndrome. Antivir Ther. 2016;21(5):455-9.

16. Omrani AS, Saad MM, Baig K, et al. Ribavirin and interferon alfa-2a for severe Middle East respiratory syndrome coronavirus infection: a retrospective cohort study. Lancet Infect Dis. 2014;14(11):1090-5.

17. Chu CM, Cheng VC, Hung IF, et al. Role of lopinavir/ritonavir in the treatment of SARS: initial virological and clinical findings. Thorax. 2004;59(3):252-6.

18. Cao B, Wang Y, Wen D, et al. A Trial of lopinavir-ritonavir in adults hospitalized with severe Covid-19. N Engl J Med. 2020. https://doi.org/10.1056/ NEJMoa2001282.

19. Mulangu S, Dodd LE, Davey RT Jr, et al. A randomized, controlled trial of ebola virus disease therapeutics. N Engl J Med. 2019;381(24):2293-303.

20. Gordon CJ, Tchesnokov EP, Feng JY, et al. The antiviral compound remdesivir potently inhibits RNA-dependent RNA polymerase from Middle East respiratory syndrome coronavirus. J Biol Chem. 2020. https://doi. org/10.1074/jbc.AC120.013056.

21. de Wit E, Feldmann F, Cronin J, et al. Prophylactic and therapeutic remdesivir (GS-5734) treatment in the rhesus macaque model of MERSCoV infection. Proc Natl Acad Sci USA. 2020. https://doi.org/10.1073/ pnas.1922083117.

22. Sheahan TP, Sims AC, Leist SR, et al. Comparative therapeutic efficacy of remdesivir and combination lopinavir, ritonavir, and interferon beta against MERS-CoV. Nat Communs. 2020;11(1):222.

23. Holshue ML, DeBolt C, Lindquist S, et al. First case of 2019 novel coronavirus in the United States. N Engl J Med. 2020;382(10):929-36.

24. Cao B. Mild/moderate 2019-nCoV remdesivir RCT_Full Text View ClinicalTrials.gov. 2020. https://clinicaltrials.gov/ct2/show/NCT04252664. Accessed 13 Feb 2020.

25. Cao B. Severe 2019-nCoV remdesivir RCT_Full text view_ClinicalTrials. gov. 2020. https://clinicaltrials.gov/ct2/show/NCT04257656. Accessed 13 Feb 2020.

26. Blaising J, Polyak SJ, Pecheur El. Arbidol as a broad-spectrum antiviral: an update. Antiviral Res. 2014;107:84-94.

27. Boriskin YS, Leneva IA, Pecheur El, et al. Arbidol: a broad-spectrum antiviral compound that blocks viral fusion. Curr Med Chem. 2008;15(10):997-1005

28. Khamitov RA, Loginova S, Shchukina VN, et al. Antiviral activity of arbidol and its derivatives against the pathogen of severe acute respiratory syndrome in the cell cultures. Vopr Virusol. 2008;53(4):9-13.

29. Lu H. Drug treatment options for the 2019-new coronavirus (2019-nCoV). Biosci Trends. 2020. https://doi.org/10.5582/bst.2020.01020.

30. Qu J. Clinical study of arbidol hydrochloride tablets in the treatment of pneumonia caused by novel coronavirus, 2020. https://clinicaltrials.gov/ ct2/show/NCT04260594. Accessed 7 Feb 2020.

31. Li W, Moore MJ, Vasilieva N, et al. Angiotensin-converting enzyme 2 is a functional receptor for the SARS coronavirus. Nature. 2003;426(6965):450-4.

32. Dimitrov DS. The secret life of ACE2 as a receptor for the SARS virus. Cell. 2003;115(6):652-3.

33. Simmons G, Reeves JD, Rennekamp AJ, et al. Characterization of severe acute respiratory syndrome-associated coronavirus (SARS-CoV) spike glycoprotein-mediated viral entry. Proc Natl Acad Sci USA. 2004;101(12):4240-5.

34. Yeung KS, Yamanaka GA, Meanwell NA. Severe acute respiratory syndrome coronavirus entry into host cells: opportunities for therapeutic intervention. Med Res Rev. 2006;26(4):414-33.

35. Yan Y, Zou Z, Sun Y, et al. Anti-malaria drug chloroquine is highly effective in treating avian influenza A H5N1 virus infection in an animal model. Cell Res. 2013;23(2):300-2.

36. Vincent MJ, Bergeron E, Benjannet $\mathrm{S}$, et al. Chloroquine is a potent inhibitor of SARS coronavirus infection and spread. Virol J. 2005;2:69.

37. Xu H, Zhong L, Deng J, et al. High expression of ACE2 receptor of 2019nCoV on the epithelial cells of oral mucosa. Int J Oral Sci. 2020;12(1):8.

38. Wang M, Cao R, Zhang L, et al. Remdesivir and chloroquine effectively inhibit the recently emerged novel coronavirus (2019-nCoV) in vitro. Cell Res. 2020. https://doi.org/10.1038/s41422-020-0282-0.

39. Gao J, Tian Z, Yang X. Breakthrough: chloroquine phosphate has shown apparent efficacy in treatment of COVID-19 associated pneumonia in clinical studies. Biosci Trends. 2020;14(1):72-3.

40. W. S. Chloroquine Prevention of Coronavirus Disease (COVID-19) in the Healthcare Setting (COPCOV). 2020. https://clinicaltrials.gov/ct2/show/ NCT04303507. Accessed 11 Mar 2020.

41. Ponticelli C, Moroni G. Hydroxychloroquine in systemic lupus erythematosus (SLE). Expert Opin Drug Saf. 2017;16(3):411-9.

42. Schrezenmeier E, Dorner T. Mechanisms of action of hydroxychloroquine and chloroquine: implications for rheumatology. Nat Rev Rheumatol. 2020;16(3):155-66.

43. Yao $X$, Ye F, Zhang $M$, et al. In vitro antiviral activity and projection of optimized dosing design of hydroxychloroquine for the treatment of severe acute respiratory syndrome Coronavirus 2 (SARS-CoV-2). Clin Infect Dis. 2020. https://doi.org/10.1093/cid/ciaa237.

44. Hernandez-Cardenas. Hydroxychloroquine Treatment for Severe COVID-19 Pulmonary Infection (HYDRA Trial) (HYDRA). 2020. https:// clinicaltrials.gov/ct2/show/NCT04315896. Accessed 20 Mar 2020.

45. Uyeki TM, Bernstein HH, Bradley JS, et al. clinical practice guidelines by the infectious diseases society of America: 2018 update on diagnosis, treatment, chemoprophylaxis, and institutional outbreak management of seasonal influenza. Clin Infect Dis. 2019;68(6):895-902.

46. Huang C, Wang Y, Li X, et al. Clinical features of patients infected with 2019 novel coronavirus in Wuhan, China. Lancet. 2020;395(10223):497-506.

47. Beigel JH, Nam HH, Adams PL, et al. Advances in respiratory virus therapeutics-A meeting report from the 6th isirv Antiviral Group conference. Antiviral Res. 2019;167:45-67.

48. Chen N, Zhou M, Dong X, et al. Epidemiological and clinical characteristics of 99 cases of 2019 novel coronavirus pneumonia in Wuhan, China: a descriptive study. Lancet. 2020;395(10223):507-13.

49. Zhao Z, Zhang F, Xu M, et al. Description and clinical treatment of an early outbreak of severe acute respiratory syndrome (SARS) in Guangzhou, P. R. China. J Med Microbiol. 2003;52(Pt 8):715-20.

50. Ho JC, Ooi GC, Mok TY, et al. High-dose pulse versus nonpulse corticosteroid regimens in severe acute respiratory syndrome. Am J Respir Crit Care Med. 2003;168(12):1449-56.

51. Thompson BT. Glucocorticoids and acute lung injury. Crit Care Med. 2003;31(4 Suppl):S253-7.

52. Ni YN, Chen G, Sun J, et al. The effect of corticosteroids on mortality of patients with influenza pneumonia: a systematic review and metaanalysis. Crit Care. 2019;23(1):99.

53. Yang JW, Fan LC, Miao XY, et al. Corticosteroids for the treatment of human infection with influenza virus: a systematic review and metaanalysis. Clin Microbiol Infect. 2015;21(10):956-63.

54. Martin-Loeches I, Lisboa T, Rhodes A, et al. Use of early corticosteroid therapy on ICU admission in patients affected by severe pandemic (H1N1)v influenza A infection. Intensive Care Med. 2011;37(2):272-83.

55. Giannella M, Alonso M, Garcia de Viedma D, et al. Prolonged viral shedding in pandemic influenza $A(\mathrm{H} 1 \mathrm{~N} 1)$ : clinical significance and viral load analysis in hospitalized patients. Clin Microbiol Infect. 2011;17(8):1160-5.

56. Wang Y, Guo Q, Yan Z, et al. Factors associated with prolonged viral shedding in patients with avian influenza $A(H 7 N 9)$ virus infection. J Infect Dis. 2018;217(11):1708-17. 
57. Arabi YM, Mandourah Y, Al-Hameed F, et al. Corticosteroid therapy for critically III patients with Middle East respiratory syndrome. Am J Respir Crit Care Med. 2018;197(6):757-67.

58. Han K, Ma H, An X, et al. Early use of glucocorticoids was a risk factor for critical disease and death from pH1N1 infection. Clin Infect Dis. 2011;53(4):326-33.

59. Matteucci C, Grelli S, Balestrieri E, et al. Thymosin alpha 1 and HIV-1: recent advances and future perspectives. Fut Microbiol. 2017;12:141-55.

60. Pica F, Gaziano R, Casalinuovo IA, et al. Serum thymosin alpha 1 levels in normal and pathological conditions. Expert Opin Biol Ther. 2018;18(sup1):13-21.

61. Wu J, Zhou L, Liu J, et al. The efficacy of thymosin alpha 1 for severe sepsis (ETASS): a multicenter, single-blind, randomized and controlled trial. Crit Care. 2013;17(1):R8

62. Gao ZC, Zhu JH, Sun Y, et al. Clinical investigation of outbreak of nosocomial severe acute respiratory syndrome. Zhongguo Wei Zhong Bing Ji Jiu Yi Xue. 2003;15(6):332-5.

63. Dawar FU, Tu J, Khattak MN, et al. Cyclophilin A: a key factor in virus replication and potential target for anti-viral therapy. Curr Issues Mol Biol. 2017:21:1-20

64. Pfefferle S, Schopf J, Kogl M, et al. The SARS-coronavirus-host interactome: identification of cyclophilins as target for pan-coronavirus inhibitors. PLoS Pathog. 2011;7(10):e1002331.

65. Morgenstern B, Michaelis M, Baer PC, et al. Ribavirin and interferon-beta synergistically inhibit SARS-associated coronavirus replication in animal and human cell lines. Biochem Biophys Res Commun. 2005;326(4):905-8.

66. Mustafa S, Balkhy H, Gabere MN. Current treatment options and the role of peptides as potential therapeutic components for Middle East Respiratory Syndrome (MERS): a review. J Infect Public Health. 2018;11(1):9-17.

67. Kuri T, Zhang X, Habjan M, et al. Interferon priming enables cells to partially overturn the SARS coronavirus-induced block in innate immune activation. J Gen Virol. 2009;90(Pt 11):2686-94.

68. Werdan K, Pilz G, Bujdoso O, et al. Score-based immunoglobulin $\mathrm{G}$ therapy of patients with sepsis: the SBITS study. Crit Care Med. 2007:35(12):2693-701.

69. Alejandria MM, Lansang MA, Dans LF, et al. Intravenous immunoglobulin for treating sepsis, severe sepsis and septic shock. Cochrane Database Syst Rev. 2013. https://doi.org/10.1002/14651858.CD001090. pub2(9):Cd001090.

70. Chen $C$, Zhang XR, Ju ZY, et al. Advances in the research of cytokine storm mechanism induced by Corona Virus Disease 2019 and the corresponding immunotherapies. Zhonghua Shao Shang Za Zhi. 2020;36:E005.

71. Hoffman E, Rahat MA, Feld J, et al. Effects of Tocilizumab, an anti-interleukin-6 receptor antibody, on serum lipid and Adipokine levels in patients with rheumatoid arthritis. Int J Mol Sci. 2019;20:18.

72. Le RQ, Li L, Yuan W, et al. FDA approval summary: tocilizumab for treatment of chimeric antigen receptor T cell-induced severe or life-threatening cytokine release syndrome. Oncologist. 2018;23(8):943-7.

73. Diagnosis and Treatment Protocol for Novel Coronavirus Pneumonia (Trial Version 7). National Health Commission \& State Administration of Traditional Chinese Medicine. 2020. http://healthcareus.sakura.ne.jp/wordp ress/2020/03/17/diagnosis-and-treatment-protocol-for-novel-coronaviru s-pneumonia-trial-version-7/. Accessed 17 Mar 2020.

74. Liu J, Manheimer E, Shi Y, et al. Chinese herbal medicine for severe acute respiratory syndrome: a systematic review and meta-analysis. J Altern Complem Med. 2004;10(6):1041-51.

75. Ji S, Bai Q, Wu X, et al. Unique synergistic antiviral effects of Shufeng Jiedu Capsule and oseltamivir in influenza A viral-induced acute exacerbation of chronic obstructive pulmonary disease. Biomed Pharmacother. 2020;121:109652.

76. Ding $Y$, Zeng $L$, Li R, et al. The Chinese prescription lianhuaqingwen capsule exerts anti-influenza activity through the inhibition of viral propagation and impacts immune function. BMC Complement Altern Med. 2017;17(1):130.

77. Du CY, Zheng KY, Bi CW, et al. Yu Ping Feng San, an ancient Chinese herbal decoction, induces gene expression of anti-viral proteins and inhibits neuraminidase activity. Phytother Res. 2015;29(5):656-61.
78. Wang C, Cao B, Liu QQ, et al. Oseltamivir compared with the Chinese traditional therapy maxingshigan-yinqiaosan in the treatment of $\mathrm{H} 1 \mathrm{~N} 1$ influenza: a randomized trial. Ann Intern Med. 2011;155(4):217-25.

79. Marano G, Vaglio S, Pupella S, et al. Convalescent plasma: new evidence for an old therapeutic tool? Blood Trans. 2016;14(2):1-6.

80. Burnouf T, Conton B, Dye JM. Convalescent plasma for Ebola virus disease N Engl J Med. 2016;374(25):2498-500.

81. Soo YO, Cheng Y, Wong R, et al. Retrospective comparison of convalescent plasma with continuing high-dose methylprednisolone treatment in SARS patients. Clin Microbiol Infect. 2004;10(7):676-8.

82. Cheng Y, Wong R, Soo YO, et al. Use of convalescent plasma therapy in SARS patients in Hong Kong. Eur J Clin Microbiol Infect Dis. 2005;24(1):44-6.

83. Lu CL, Murakowski DK, Bournazos S, et al. Enhanced clearance of HIV1 -infected cells by broadly neutralizing antibodies against HIV-1 in vivo. Science. 2016;352(6288):1001-4.

84. Zhao J, Perera RA, Kayali G, et al. Passive immunotherapy with dromedary immune serum in an experimental animal model for Middle East respiratory syndrome coronavirus infection. J Virol. 2015;89(11):6117-20.

85. Chen L, Xiong J, Bao L, et al. Convalescent plasma as a potential therapy for COVID-19. Lancet Infect Dis. 2020. https://doi.org/10.1016/s1473 -3099(20)30141-9.

86. Fowler RA, Lapinsky SE, Hallett D, et al. Critically ill patients with severe acute respiratory syndrome. JAMA. 2003;290(3):367-73.

87. Kumar A, Zarychanski R, Pinto R, et al. Critically ill patients with 2009 influenza A(H1N1) infection in Canada. JAMA. 2009;302(17):1872-9.

88. Rochwerg B, Brochard L, Elliott MW, et al. Official ERS/ATS clinical practice guidelines: noninvasive ventilation for acute respiratory failure. Eur Respir J. 2017;50:2.

89. Frat JP, Thille AW, Mercat A, et al. High-flow oxygen through nasal cannula in acute hypoxemic respiratory failure. N Engl J Med. 2015;372(23):2185-96.

90. Hui DS, Chow BK, Lo T, et al. Exhaled air dispersion during high-flow nasal cannula therapy versus CPAP via different masks. Eur Respir J. 2019;53:4.

91. Hui DS, Chow BK, Chu L, et al. Exhaled air dispersion during coughing with and without wearing a surgical or N95 mask. PLoS ONE. 2012;7(12):e50845.

92. Society RccoCT. Expert consensus on preventing nosocomial transmission during respiratory care for critically ill patients infected by 2019 novel coronavirus pneumonia. Zhonghua Jie He He Hu Xi Za Zhi. 2020;17:E020

93. Alhazzani W, Moller MH, Arabi YM, et al. Surviving Sepsis Campaign: guidelines on the management of critically ill adults with Coronavirus Disease 2019 (COVID-19). Intensive Care Med. 2020. https://doi. org/10.1007/s00134-020-06022-5.

94. Guérin C, Reignier J, Richard J-C, et al. Prone positioning in severe acute respiratory distress syndrome. N Engl J Med. 2013;368(23):2159-68.

95. $X u Y$, Deng $X$, Han $Y$, et al. A multicenter retrospective review of prone position ventilation (PPV) in treatment of severe human H7N9 Avian flu. PLOS ONE. 2015;10(8):e0136520.

96. Noah MA, Peek GJ, Finney SJ, et al. Referral to an extracorporeal membrane oxygenation center and mortality among patients with severe 2009 influenza A(H1N1). JAMA. 2011;306(15):1659-68.

97. Alshahrani MS, Sindi A, Alshamsi F, et al. Extracorporeal membrane oxygenation for severe Middle East respiratory syndrome coronavirus. Ann Intensive Care. 2018;8(1):3.

98. MacLaren G, Fisher D, Brodie D. Preparing for the most critically ill patients with COVID-19: the potential role of extracorporeal membrane oxygenation. JAMA. 2020. https://doi.org/10.1001/jama.2020.2342.

99. Poston JT, Patel BK, Davis AM. Management of critically ill adults with COVID-19. JAMA. 2020. https://doi.org/10.1001/jama.2020.4914.

\section{Publisher's Note}

Springer Nature remains neutral with regard to jurisdictional claims in published maps and institutional affiliations. 